\title{
Eine Bilanz der \\ Pflegeversicherung in 6 Thesen
}

Die unmittelbare Betroffenheit von dem Lebensrisiko Pflegebedürftigkeit steigt. Die Menschen erwarten nicht nur fachliche und verlässliche, sondern auch verständliche Lösungen. Im folgenden wird eine Zwischenbilanz der vor ihrer Reform stehenden Pflegeversicherung gezogen.

\section{Gerhard Naegele}

1. Zum Zeitpunkt der Drucklegung dieser Thesen befindet sich die in der Koalitionsvereinbarung angekündigte Reform der Pflegeversicherung am Beginn der Gesetzgebungsphase. Ganz sicher erwartet werden können die Dynamisierung der Leistungen, die Ausweitung der Leistungen nach dem Pflegeleistungsergänzungsgesetz, die Stärkung der geriatrischen Rehabilitation, die Erweiterung des Haushaltsbegriffs, wodurch die Förderung von Haushaltsgemeinschaften eingeschlossen wäre, der Ausbau der Palliativversorgung, die Erweiterung der Möglichkeiten der integrierten Versorgung, Verbesserungen in der Qualitätssicherung auch in der Pflege, eine bessere Vernetzung der Leistungen der Pflegeversicherung untereinander sowie ein besseres Versorgungsmanagement insbesondere nach Krankenhausaufenthalt. Zudem soll „mittelfristig" der Pflegebegriff überarbeitet und ein darauf bezogenes erweitertes Begutachtungsverfahren entwickelt werden. Ob und inwieweit die anstehende Novelle des SGB XI aber auch tatsächlich zu einer „echten“ Strukturreform wird, bleibt abzuwarten - Grund genug, in einer Art sozialpolitischer Zwischenbilanz Erfolg zu ziehen und einige zentrale Reformerfordernisse aufzuzeigen.

2. Die Pflegeversicherung ist und war die einzig „richtige“ Antwort auf die pflegebedingten Herausforderungen einer insgesamt alternden Gesellschaft. Eine positive Würdigung schließt jedoch fachlichen Veränderungs- und Neugestaltungsbedarf nicht aus („Erfolgsmodell mit Reformbedarf") (s.u. Pkte. 4, 5).

3. Insgesamt ist und war die Pflegeversicherung vor allem aus folgenden Gründen ein „sozialpolitischer Erfolg“:

- Pflegebedürftigkeit hat damit als eigenständiges Lebensrisiko und als soziales (und eben nicht privates)

Prof. Dr. Gerhard Naegele, Institut für Gerontologie an der Universität Dortmund (www.uni-dortmund.de/FFG)
Problem nach rd. 25 Jahren Vorklärungen (endlich) die erforderliche öffentliche Anerkennung gefunden.

- Gleichzeitig ist das Pflegebedürftigkeitsrisiko einer der Tradition der deutschen Sozialpolitik angemessenen Absicherungsform (,eigenständige Säule im Sozialversicherungssystem“) zugeführt worden.

- Auch konnte das Pflegerisiko von dem Ruch der „Armenfürsorge" und viele Pflegebedürftige von der Sozialhilfeabhängigkeit befreit werden.

- Allerdings gilt auch, dass die mit dem SGB XI gefundene Lösung - historisch ebenfalls einmalig - mit einem ehernen Konstruktionsprinzip der Sozialversicherung gebrochen hat, in dem nämlich bei der Finanzierung die Arbeitgeberbeiträge zu Lasten der BeitragszahlerInnen kompensiert wurden.

- Die Pflegeversicherung hat vielen privaten Pflegepersonen eine (wenn auch bescheidene) soziale Sicherung gebracht.

- Die Pflegeversicherung hat einen längst überfälligen Beitrag zur Offenlegung und zugleich Rationalisierung der professionellen „Pflegewelt" geleistet.

- Die häusliche Pflege und das häusliche Umfeld sind strategisch zum Bezugspunkt sozialpolitischer Bemühungen avanciert.

- Die bestehende Pflegeinfrastruktur konnte nachhaltig ausgeweitet werden, bestimmte „Engpasssituationen“ (z.B. Tagespflege, Pflege an Wochenenden) sind überwunden. Zudem gibt es ganz erhebliche positive Arbeitsmarkteffekte.

- Der „Pflegemarkt“ ist insgesamt pluraler geworden, auf Anbieterseite gibt es ein Mehr an Wettbewerb.

- Fachwissenschaftlich ist anzumerken, dass der zuvor inferiore Stellenwert der Pflege gegenüber der Medizin gestärkt worden ist.

- Professionalisierung und Verwissenschaftlichung der Pflege wurden gefördert und weiter vorangetrieben.

- Die im Ausland längst eingeführte Debatte um Verbraucherschutz und Kundenbeteiligung („,user involve- 
ment"; „user empowerment") in der Pflege ist nun auch nach Deutschland gelangt.

- Aus sozialpolitischer Sicht ist die neue „Kundenrolle“ wenn auch nicht überall eingeübt oder gar möglich zweifellos positiver zu bewerten als die des/r „dankbaren Nehmers/in" früherer Jahre.

- Die Pflegeversicherung hat wesentliche Anstöße für die in der Pflege - gegenüber der Medizin - längst überfällige Qualitätssicherungsdiskussion gegeben.

- Sie hat schließlich die Sozialhilfeabhängigkeit vieler Heimbewohner und die Sozialhilfe-Kosten-Belastung der Kommunen durch Pflegebedürftigkeit nachhaltig gesenkt.

4. Diesen Erfolgen steht eine Reihe von teilweise bereits bei der Einführung von Fachleuten kritisierten „Schwachstellen“ gegenüber. Zu den wichtigsten zählen:

- das von Anfang an unter dem finanziellen Aspekt der Begrenzung des leistungsberechtigten Personenkreises konzipierte, zu enge verrichtungsbezogene Konzept von Pflegebedürftigkeit;

- die dauerhaft erwartbare und in der Zwischenzeit auch eingetretene reale Leistungsentwertung der Zahlbeträge wegen fehlender Dynamisierung;

- das politisch - vermutlich ebenfalls aus Finanzgründen - gewollte Primat der Geldleistungen und der deutlich stärker als erwartet stattgefundene „Run“ der Leistungsberechtigten darauf, der zu erheblichen Mitnahmeeffekten geführt hat und dessen Auswirkungen auf die häusliche Pflegequalität höchst strittig ist. Nur wenig bekannt ist, dass mit der Geldleistungsoption die Bundesrepublik im internationalen Vergleich nahezu einmalig dasteht und im fachwissenschaftlichen Diskurs nicht selten Erstaunen bis Kopfschütteln hervorruft.

- „Angehörigenorientierung“ und häuslich-familiäre Pflege, die beide in Anbetracht des demografischen und sozialen Wandels („Singularisierung“ des Alters in Verbindung mit Hochaltrigkeit) einerseits und prognostizierter immer schwerwiegender und immer multikomplexer werdenden Fälle von Pflegebedürftigkeit andererseits zwangsläufig Begründungs- und Konstruktionsprobleme erkennen lassen.

- die starre Trennung zwischen ambulanten und stationären Pflegeleistungen, wodurch u.a. individuell zugeschnittene Hilfen für die immer komplexer werdenden Bedarfs- und Nachfragesituationen einschließlich neuer Wohn- und Pflegeformen erschwert werden.

- Mängel in der Umsetzung des Primats „Prävention und Rehabilitation vor Pflege".

- die unzureichend institutionalisierte, unabhängige Absicherung der Qualität, die in der Gesundheitspolitik fast widerstandslos akzeptiert worden ist, aber für die Pflege vielen immer noch keine wichtige politische Gestaltungsaufgabe zu sein scheint.
- Probleme und Friktionen in der Kooperation und Koordination zwischen den an der professionellen Pflege beteiligten Leistungsbereichen, Akteursgruppen und Berufen mit der Folge vielfältiger Fragmentierungen in der Versorgung kranker und pflegebedürftiger Menschen.

5. Ausgehend davon und nach Sichtung der aktuellen sozialpolitischen Debatte um die Reform der Pflegeversicherung lässt sich folgende Liste von Reformschwerpunkten benennen, die zugleich die Funktion von Prüfsteinen für die anstehende Reform haben könnte:

- Weiterentwicklung des Pflegebedürftigkeitsbegriffs und -konzeptes insbesondere in Richtung auf Öffnung für den besonderen Pflege- und Betreuungsbedarf von Menschen mit demenzbedingten Fähigkeitseinschränkungen, geistigen Behinderungen und psychischen Erkrankungen. In der Konsequenz erfordert dies die Überwindung des streng verrichtungsbezogenen Einstufungsverfahrens zugunsten eines erweiterten Begutachtungsverfahrens, das nicht nur die psychischen Dimensionen, sondern auch die Förderung und Nutzung vorhandener Präventionspotenziale sowie die Erschließung weiterer Leistungen einbeziehen, im Idealfall somit zugleich auf die Optimierung des Pflegearrangements zielen sollte. Bekanntlich arbeitet derzeit eine namhafte Kommission daran, es ist jedoch offen, ob deren Ergebnisse, was zu wünschen wäre, noch Eingang finden in das laufende Gesetzgebungsverfahren oder davon abgekoppelt werden - mit der dann problematischen und insbesondere in der Fachöffentlichkeit schwer zu kommunizierenden Folge einer weiteren zeitlichen Verzögerung bei der schon seit langem unisono eingeforderten Neukonzeption des Pflegebegriffs.

- Pflegebedürftige sind in der Regel auch behinderte Menschen, für die daher auch die Zielsetzungen und Maßgaben des SGB XI gelten, hier insbesondere des sozialen Teilhabeprinzips. An dem in Behindertenpolitik und Behindertenrecht längst eingeführten Paradigmen der sozialen Teilhabesicherung und der Selbstbestimmung der Pflegebedürftigen könnten sich künftig auch in der Pflege alle Leistungen und alle Reformvorhaben orientieren.

- Dynamisierung der Leistungen, um den laufenden Wertverfall der Pflegeleistungen und die dadurch bedingte problematische Verschiebung in Richtung auf die Inanspruchnahme von Sozialhilfeleistungen endlich zu stoppen. Die Regeldynamisierung ist ein „Muss" für die anstehende Reform und sollte - schon um entsprechende positive Signale in der Öffentlichkeit zu setzen - nicht unter irgendeinem Finanzierungsvorbehalt kommuniziert werden.

- Speziell aus der Perspektive sonst wieder drohender Sozialhilfesteigerungen gilt es vor der als eine realistische Option angekündigten Umschichtung der Geldströme („Neujustierung“) - weg von der stationären und hin 
zur ambulanten Pflege - zu warnen; auch deswegen, um eine neue Armutsdebatte bei stationärer Pflegebedürftigkeit und damit die Gefahr eines Reputationsverlustes der Pflegeversicherung zu vermeiden, welche die aktuelle Reformdebatte nur unnötig erschweren würde.

- Überwindung des zu engen Leistungskatalogs im SGB XI und mehr Differenzierung bei den Pflegestufen, um mehr Raum für personenbezogene und situationsangemessene Bedarfslagen zu erhalten. Nur so ließen sich innovative und flexible Zwischenformen zwischen ambulant und stationär oder neue Versorgungsformen zwischen Wohnen und Pflege finanzieren bzw. nur dadurch werden zu ihrem Auf- und Ausbau angemessene ökonomische Anreizstrukturen geschaffen. Von einer Reform der Pflegeversicherung muss unbedingt ein Signal für neue Versorgungs- und Wohnformen und zugunsten einer Deinstitutionalisierung der Versorgung - wo immer auch möglich - ausgehen.

- Ausweitung von bedarfsgerechten, ambulanten pflegeinfrastrukturellen Maßnahmen zur Stärkung ebenso wie zur Qualifizierung der familiären Pflege. Neben einer professionell organisierten pflegefachlichen Begleitung der pflegenden Familien zählt dazu eine unabhängige Pflegeberatung. Hier sind insbesondere die Kommunen im Rahmen ihres ihnen verfassungsrechtlich zugewiesenen sozialen Daseinsvorsorgeauftrages gefordert. Pflegepolitik ist somit immer auch Teil von kommunaler Familienpolitik.

- Wirksame Unterstützung von berufstätigen Pflegepersonen - aus meiner Sicht möglichst mit betriebsnahen und/oder tarifvertraglichen Lösungen. Der immer wieder ins Gespräch gebrachte Pflegeurlaub - auf den ersten Blick naheliegend - könnte sich beim zweiten Blick als psychosozialer, beschäftigungspolitischerund sicherungsmäßiger „Bumerang“ für die davon zumeist betroffenen Frauen erweisen.

- Kritische Überprüfung des Konzeptes der Geldleistungen, nicht weil ich der in ihm unterstellten Selbstbestimmungsfähigkeit misstraue, sondern aus Gründen der Sicherung der häuslichen Pflegequalität. Zumindest für eine stärkere Kontrolle der zweckentsprechenden Verwendung des Pflegegeldes sollte gesorgt werden. Gerade in der häuslichen Pflege - geleistet primär unter den Anreizstrukturen des Pflegegeldes gilt es auf Pflegefachlichkeit zu achten.

- Flexibilisierung des Sachleistungsprinzips durch Gewährung von personenbezogenen, frei verfügbaren Budgets, d.h. auf einen kurzen Nenner gebracht: "Geldleistung zum Sachleistungseinkauf"; allerdings nur unter der Voraussetzung der Schaffung von Assistenzmodellen bzw. „care manager“, um Mitnahmeeffekte oder neue Formen der Schwarzarbeit zu vermeiden. Vorbilder gibt es bekanntlich bereits im SGB IX und mit den sich gerade in der Erprobungsphase befindlichen Pflegebudgets.

- Schaffung von verbindlichen, von Kosten- und Leistungsträgern gleichermaßen getragenen Versorgungs- standards und Qualitätskriterien. Dieses Anliegen sollte eingebunden sein in die übergeordnete Forderung nach Institutionalisierung der verschiedenen, bislang weitgehend nebeneinander herlaufenden Strängen der Qualitätssicherung - nach meinen Vorstellungen möglichst in Gestalt einer unabhängigen Institution - wobei hier an die entsprechenden strukturellen Vorläufer und Fortschritte in der Gesundheitsreform angeknüpft werden könnte.

- Verbesserung der Pflege- und Arbeitsbedingungen in den Heimen, auf die auch künftig nicht verzichtet werden kann, wenn auch in Anbetracht des offensichtlichen „Strukturwandels der HeimbewohnerInnen“ ganz sicher anders als heute konzeptualisiert. Eine wichtige Voraussetzung dafür ist neben einer klaren Leistungsbeschreibung und eindeutiger Qualitätskriterien vor allem im Bereich von Struktur- und Ergebnisqualität die Implementierung von geeigneten Systemen der Personalbemessung. Das gerade vor dem Abschluss stehende NRW-Projekt „Referenzmodelle“ lässt für alle diese Anforderungen fruchtbare Erkenntnisse erwarten.

- Flexible Ausgestaltung des Pflegeversicherungsrechtes mit dem Ziel der Überwindung der Mängel in der systemimmanenten Steuerungslogik, u.a. durch möglichst systemübergreifende Verzahnungen von Leistungsbereichen, Akteurs- und Berufsgruppen einschließlich des Verhältnisses von Professionellen und Laien aus dem familiären Umfeld oder dem bürgerschaftlichen Engagement. Letztlich geht es um die Einbeziehung der Pflege in ein integriertes Versorgungssystem.

- Nachhaltige Sicherstellung von professionellem, an verschiedenen Einsatzorten in der Pflege tätigem Personal unterschiedlicher Qualifikationen. In quantitativer Hinsicht ist an Neurekrutierung (bei Bedarf auch aus dem Ausland) ebenso wie an Maßnahmen des Personalerhalts (z.B. durch Maßnahmen zur Humanisierung des Arbeitsbelebens bzw. der Verbesserung der Arbeitsbedingungen in der Pflege) zu denken. Zugleich aber gilt es, einen neuen „Schwarzmarkt" mit prekären Arbeits- und Beschäftigungsverhältnissen durch wirksame Formen der Regulierung zu vermeiden. In qualitativer Hinsicht muss es künftig stärker um eine fachlich begründete Spreizung bei den Qualifikationsanforderungen und Tätigkeitsprofilen - entsprechend unterschiedlicher Bedarfe in unterschiedlichen Pflegeorten - gehen.

6. In der Bewältigung der Pflegebedürftigkeit liegt eine der zentralen gesellschaftlichen und kulturellen Herausforderungen der nächsten Jahrzehnte. Die Menschen in diesem Lande erwarten darauf bezogene verlässliche, verständliche und für sie nachvollziehbare Antworten. Die hiermit konturierten Prüfsteine für nachhaltige Reformen sind in Teilen voraussetzungsvoll und ganz gewiss nicht „umsonst“ zu haben.

- Gefordert ist zunächst eine eindeutig positive Grundeinstellung in der Bevölkerung und in der Politik für die 
Selbstverständlichkeit von angemessener Qualität auch in der Pflege und zu fachlich-qualitativen Veränderungs- und Neugestaltungserfordernissen im SGB XI. Dies betrifft insbesondere solche Maßnahmen, die mehr finanziellen Einsatz fordern. Es muss eine ehrliche Auseinandersetzung darüber geführt werden, was wir - als Gesellschaft, aber auch als Einzelne - für eine fachlich gute professionelle Pflege zu investieren bereit sind. Hierzu sollte man übrigens auch von politischen Entscheidungsträgern Mut und Ehrlichkeit erwarten, und zwar jenseits der politischen Ziele von Ausgabeneinschränkungen und Gesamtbeitragssatzstabilität. Bei allem Verständnis für die Forderung nach mehr Eigenverantwortlichkeit der BürgerInnen und mehr Mitverantwortung der Familien oder aus dem bürgerschaftlichen Engagement, muss es bei der öffentlichen Grundverantwortlichkeit für die Daseinsvorsorge auf dem Gebiet der Pflege bleiben, zumal noch bei stetig wachsender unmittelbarer und mittelbarer Betroffenheit.

- Es führt kein Weg an der Erkenntnis vorbei, dass für eine gute Pflege und Betreuung in einer insgesamt alternden Gesellschaft mehr Geld aufgebracht werden muss, allerdings nicht von Seiten der BeitragszahlerInnen allein, aber eben auch von diesen. Es ist kein Geheimnis, dass ich mich in diesem Zusammenhang schon immer als Vertreter der Bürgerversicherung geoutet habe, wohl wissend um bestehende politische Durchsetzungsschwierigkeiten. Eine moderate Beitragssatzanhebung ist mittelfristig und dauerhaft unausweichlich. Allerdings gilt auch, dass die Probleme einer alternden Gesellschaft keine sind, die sich allein über den Weg der Sozialversicherung lösen lassen. Auch von daher plädiere ich für einen Finanzierungs-Mix, unter Einbezug einer Beteiligung des Bundes, von Bürgerversicherungselementen und ggfs. auch einer weiteren moderaten Zusatzbelastung der RentnerInnen. Zusätzliche Finanzmittel lassen sich darüber hinaus durch den bereits im Koalitionsvertrag vereinbarten Finanzausgleich zwischen der gesetzlichen und der privaten Pflegeversicherung aktivieren. Die Kapitaldeckungsoption kann immer nur eine zusätzliche Option sein, allerdings mit dem positiven pädagogischen Nebeneffekt eines Beitrages zur individuellen und öffentlichen Bewusstwerdung der finanziellen Implika- tionen der Pflegeproblematik. Sie muss zudem vor politisch motivierten Zugriffen geschützt werden.

- Auf jeden Fall aber gilt es zu verhindern, dass Finanzierungsprobleme der Pflegeversicherung dazu führen, dass dadurch die erforderliche fachliche Reformdebatte weniger ernst genommen oder gar ganz verdrängt wird.

- Andererseits wiederum darf nicht der Eindruck entstehen, ein Mehr an Geld im System käme bei den betroffenen Menschen gar nicht an. Voraussetzung für eine höhere Leistungsbereitschaft der Versicherten ist ein höheres Vertrauen in die Qualität und Leistungsstrukturen der Anbieter. Dazu bedarf es nicht zuletzt auf dieser Seite ein Mehr an Bemühungen, aber auch mehr Verbraucherschutz und vor allem, da auch in der Pflege nicht an die Selbstheilungskräfte des Marktes geglaubt werden darf, einer sehr viel stärker ergebnisorientierten Qualitätssicherungspolitik.

- Auch die politische Zuständigkeit für die Zukunft der Pflege muss neu diskutiert werden. Wenngleich immer wieder implizit eine Bundeszuständigkeit eingefordert wird, sie allein löst die unterschiedlichen Herausforderungen nicht. Zu Recht spricht das SGB XI von der Pflege als einer gesamtgesellschaftlichen Aufgabe. Die gerade vollzogene Föderalisierung des Heimrechtes hat bekanntlich die Länder und die Kommunen stärker in die Verantwortung genommen. Dass vor allem die Kommunen ihre Rolle in der Pflegepolitik revitalisieren müssen, ist eine von vielen ExpertInnen seit langem unterstützte Forderung und gilt hier nachhaltig zu unterstreichen. Auffällig ist aber auch, dass neben den Kommunen hierzulande auch die Betriebe und die Tarifvertragsparteien in der Pflege zu wenig engagiert sind bzw. keine Mitzuständigkeit sehen.

- Zum Schluss: Es geht insgesamt um die Erhaltung des öffentlichen Ansehens der Pflegeversicherung in der Bevölkerung. Die unmittelbare und mittelbare Betroffenheit von diesem neuen Lebensrisiko steigt. Die Menschen erwarten nicht nur fachliche und verlässliche, sondern auch verständliche Lösungen. Es gilt dabei, Menschen in ohnehin schon stark problematischen Lebenslagen nicht noch durch zusätzliche unverständliche oder gar überbürokratisierte Lösungen noch weiter zu verunsichern. 